\title{
Sustainability in International Aid Programs; Identification of Working Concepts of Sustainability and Its Contributing Factors
}

\author{
Hyejin Lee ${ }^{1}$ \\ ${ }^{1}$ Department of Urban and Environmental Policy and Planning, Tufts University, 97 Talbot Avenue, Medford, \\ Massachusetts, USA \\ Correspondence: Hyejin Lee, Department of Urban and Environmental Policy and Planning, Tufts University, 97 Talbot \\ Avenue, Medford, Massachusetts, USA.
}

Received: November 29, 2016

Accepted: December 6, 2016

Available online: December 8, 2016

doi:10.11114/ijsss.v5i1.2055

URL: http://dx.doi.org/10.11114/ijsss.v5i1.2055

\begin{abstract}
An international aid program is a process that takes complex strategic planning with persistent collective efforts. Although dependent on a program's nature, many international aid programs seek sustained benefits and services as their eventual goal after a donor funding ceases. Accordingly, sustainability of international aid programs has been much discussed for aid effectiveness and efficiency. Despite its well-recognized importance, and due to its complexity, the term 'sustainability' has yet to be clearly conceptualized across even similar programs. Additionally, there seems to be a lack of consensus on what common factors may contribute to sustainability. The current study reviewed 16 select papers of international aid programs related to health, food or rural development, and identified commonly utilized working concepts of sustainability and its key factors. The most cited concept of sustainability in the select papers was sustained delivery of program services and outcomes. For its contributing factors, eleven factors were identified in the order of frequency: capacity building (16), political commitment (10), continuous funding resource (8), community participation (6), linkage or connectedness (5), acceptance in socio-culture (5), program effectiveness (5), institutionalization (3), transition of responsibility (3), negotiation (3) and communication (3). The findings can help plan more sustainable programs in relevant fields.
\end{abstract}

Key words: sustainability, factors, sustained benefit, continued impact, aid program, international development

\section{Introduction}

Sustainability has been at the heart of the debate around aid effectiveness and efficiency since the 1980s (Brown, 1998). As a sign of its importance to donors, sustainability is one of the five measures by which development interventions are evaluated, the other four being relevance, efficiency, effectiveness and impact (Brown, 1998). As development programs are a process that takes strategic planning with persistent collective efforts, there are numerous potential factors that affect sustainability at any point during the program life cycle (McConville \& Mihelcic, 2007). A responsible program, thus, is one that respects the complexity of this process to ensure sustainability (McConville \& Mihelcic, 2007). Most concerns about sustainability are related to premature discontinuation of programs after the initial period of support; rapid erosion of program achievements with funding discontinuation wastes human, monetary, and technical investments, and can diminish community trust and support for future initiatives (Gruen et al., 2008).

However, incorporating sustainability into program design, implementation, monitoring and evaluation is difficult. To begin with, vague concepts of sustainability hinder devising methods to design and implement sustainable programs, and to assess what has been sustained after the program ends (Rogers \& Coates, 2015). Stirman et al. (2012) pointed out lack of a working definition of sustainability limits research on sustainability because its conceptualization has important implications on how it is investigated (Stirman et al., 2012). The Organization for Economic Cooperation and Development (OECD), for instance, defines sustainability as 'survival of projects and programs after an initial period of investment'(OECD, 1989). Stirman et al. (2012) disputed the OECD's broad definition can render results difficult to interpret, especially when some aspects of a program continued while others did not (Stirman et al., 2012). Nevertheless, there are few evidence-based models or indicators to assess sustainability, partly due to heterogeneous natures of programs' objectives and designs, along with little consensus around generally relevant preconditions for sustainability (Gruen et al., 2008). 
Despite challenges to study sustainability, a growing body of literature from a wide range of sectors has sought to identify useful definitions or concepts of program sustainability and factors that are likely to be important for ensuring sustained benefits. Literature covering sustainability in international aid programs with health, food, or rural development varies from solely theory-basis to empirical case study. Thus, to better understand sustainability and its factors, the current study attempts to achieve two objectives: first, to identify working concepts of sustainability across select literature and second, to quantify factors that may contribute to sustainability. The findings of this study intend to help formulate more effective and efficient strategies for program planners and policy-makers to sustain program benefits in international health, food or rural development aid.

\section{Methods}

This study adopted a standardized methodology for a systematic review (Hagen-Zanker \& Mallet, 2013; Pallas et al., 2013). The first phase specified research questions, key words and relevant search databases. This phase also established inclusion and exclusion criteria, and pilot-tested the select key words and search databases not to retrieve too many irrelevant or too few results. The search words included, but were not limited to, the most commonly used synonyms: sustainability framework, model, matrix, factors, transition, program continuation, community-based, rural development, international aid, healthcare, food programs or their combinations. The search databases were Google Scholar, Econlit, PubMed and Development Experience Clearinghouse for peer-reviewed literature published up to the year of 2016. Gray literature published by authoritative development organizations or research institutions was included. The review process excluded search returns of sustainability on natural environment, agriculture, ecosystem or climate change, and programs implemented in developed countries. Two rounds of the screening retrieved the most relevant studies; the first screening focused on titles and abstracts, and the second screening reviewed 25 papers in their full texts and details, which reduced to final 16 papers. The 16 papers were analyzed to determine their working concepts of sustainability and factors that were identified as preconditions, external settings, or characteristics of sustained programs. Table 1 summarizes the methodologies adopted by the 16 papers.

Table 1. Methods adopted by the select papers for sustainability framework and factors

\begin{tabular}{|c|c|c|c|c|c|}
\hline \multirow[b]{2}{*}{ Publication * } & \multicolumn{5}{|c|}{ Methods } \\
\hline & $\begin{array}{l}\text { Literature } \\
\text { review }{ }^{1}\end{array}$ & Interview $^{2}$ & $\begin{array}{l}\text { Case study } \\
\text { analysis }\end{array}$ & $\begin{array}{c}\text { Document } \\
\text { review }{ }^{4}\end{array}$ & $\begin{array}{c}\text { Author } \\
\text { experience }^{5}\end{array}$ \\
\hline Bao et al., 2015 & $\mathrm{O}$ & $\mathrm{O}$ & & & $\mathrm{O}$ \\
\hline Bennett et al., 2011 & $\mathrm{O}$ & $\mathrm{O}$ & & $\mathrm{O}$ & \\
\hline Bossert, 1990 & $\mathrm{O}$ & & $\mathrm{O}$ & & \\
\hline Brinkerhoff \& Goldsmith, 1992 & $\mathrm{O}$ & & $\mathrm{O}$ & & \\
\hline Brinkerhoff, 1994 & $\mathrm{O}$ & & $\mathrm{O}$ & & \\
\hline Brown, 1998 & $\mathrm{O}$ & & & & \\
\hline Chilundo et al., 2015 & $\mathrm{O}$ & $\mathrm{O}$ & $\mathrm{O}$ & $\mathrm{O}$ & \\
\hline Esselman, 1994 & $\mathrm{O}$ & & $\mathrm{O}$ & & \\
\hline Gruen et al., 2008 & $\mathrm{O}$ & & & & \\
\hline Hanh et al., 2009 & $\mathrm{O}$ & & & & \\
\hline Levinger \& McLeod, 2002 & $\mathrm{O}$ & $\mathrm{O}$ & $\mathrm{O}$ & & \\
\hline Mog, 2004 & $\mathrm{O}$ & & & & \\
\hline Pallas et al., 2013 & $\mathrm{O}$ & & & & \\
\hline Rasschaert et al., 2014 & $\mathrm{O}$ & $\mathrm{O}$ & & & \\
\hline Sarriot et al., 2004 & $\mathrm{O}$ & $\mathrm{O}$ & & & \\
\hline Walsh et al., 2012 & $\mathrm{O}$ & $\mathrm{O}$ & $\mathrm{O}$ & & \\
\hline
\end{tabular}

*: Alphabetical order of first author's last name: if the same, papers in the order of year

${ }^{1}$ : Including both systemic and non-systemic reviews

${ }^{2}$ : Key-informants, stakeholders, relevant officials and field experts etc.

${ }^{3}$ : Using completed programs or ex-post evaluation results as examples of applicable theories, or as empirical evidence for building frameworks and identifying factors

${ }_{5}^{4}$ : Documents other than peer-reviewed papers such as program internal documents

5 : Reflections on professional experiences

Then, identified concepts of sustainability described in each paper were categorized according to their perceived similarities in meaning. For sustainability factors, the select papers tended to use different terms: components, elements, determinants, criteria, domains as well as factors. For consistency, the term 'factor' is used throughout this paper unless otherwise noted. The number of times a given factor was identified in each paper was quantified for the factor analysis. The following sections present and discuss the findings. 


\section{Concepts of Sustainability}

Table 2 summarizes three main concepts of sustainability identified in the review. Overall, the most prevalent concept of sustainability is sustained delivery of program services and outcomes over time. This is partly in line with one of the earliest definitions of sustainability by Honadle and Van Sant (1985), which is 'continuation of benefit flows with or without the programs or organizations that stimulated those benefits in the first place' (Honadle \& VanSant, 1985). Three papers identified institutional capacity, survival or longevity as fundamental to sustainability. Institutional survival, as stated in Brinkerhoff and Goldsmith (1992), is 'ability of an organization to produce outputs of sufficient value, thus it acquires enough inputs to continue production at a steady or growing rate'. In other words, a sustainable institution earns the adherence of a sufficient body of people to receive continuous support it needs (Brinkerhoff \& Goldsmith, 1992). Gruen et al. (2008) emphasized institutional survival for measuring continued delivery of program benefits and a level of program institutionalization in organizations or social systems (Gruen et al., 2008). This concept is connected to the first concept in that institutional survival can be part of a condition to sustain benefit flows. Five papers adopted a multidimensional dynamic nature of sustainability stressing its inherent difficulty to simplify meanings and applications of sustainability (Mog, 2004). These five papers emphasized an evolving concept of sustainability with interactive and changing aspects of stakeholders, components, external or contextual environments of programs. One paper by Levinger and McLeod (2002) provided no specific concept of sustainability, yet the authors mentioned on sustainability 'where a development service no longer needs to be provided, because the change the service was designed to promote has now been fully internalized by the targeted population' (Levinger \& McLeod, 2002). This is due to their focus area, food-assisted education programs; once the target population adopts the promoted behavior changes, the program achieves its goal.

Table 2. Working concepts of sustainability and counts of appearance across select papers

\begin{tabular}{|c|c|c|c|}
\hline \multirow[b]{2}{*}{ Publication * } & \multicolumn{3}{|c|}{ Working concept of sustainability ${ }^{1}$} \\
\hline & $\begin{array}{l}\text { Sustained delivery of } \\
\text { program services and } \\
\text { outcomes }\end{array}$ & $\begin{array}{c}\text { Not static but evolving; stressing } \\
\text { interactive aspects of stakeholder, } \\
\text { program components, external } \\
\text { environment }\end{array}$ & $\begin{array}{c}\text { Institutional } \\
\text { capacity, survival or } \\
\text { longevity }^{2}\end{array}$ \\
\hline Bao et al., 2015 & $\mathrm{O}$ & & \\
\hline Bennett et al., 2011 & $\mathrm{O}$ & & \\
\hline Bossert, 1990 & $\mathrm{O}$ & & $\mathrm{O}$ \\
\hline Brinkerhoff \& Goldsmith, 1992 & $\mathrm{O}$ & & $\mathrm{O}$ \\
\hline Brinkerhoff, 1994 & $\mathrm{O}$ & & \\
\hline Brown, 1998 & $\mathrm{O}$ & & $\mathrm{O}$ \\
\hline Chilundo et al., 2015 & & $\mathrm{O}$ & \\
\hline Esselman, 1994 & $\mathrm{O}$ & & \\
\hline Gruen et al., 2008 & & $\mathrm{O}$ & \\
\hline Hanh et al., 2009 & $\mathrm{O}$ & & \\
\hline \multicolumn{4}{|l|}{ Levinger \& McLeod, $2002^{3}$} \\
\hline Mog, 2004 & & $\mathrm{O}$ & \\
\hline Pallas et al., 2013 & $\mathrm{O}$ & & \\
\hline Rasschaert et al., 2014 & $\mathrm{O}$ & & \\
\hline Sarriot et al., 2004 & & $\mathrm{O}$ & \\
\hline Walsh et al., 2012 & & $\mathrm{O}$ & \\
\hline
\end{tabular}

*: Alphabetical order of first author's last name: if the same, papers in the order of year

1: Most explicit and central concept in each paper was cited; three papers equally weighed concept 1 and 3

2 : Long-term institution survival for continuous service delivery after program completion

3: No specific concept provided

While the overarching concepts of sustainability are grouped based on their primary emphasis, there are nuanced differences that vary according to specifics of programs or intended usages of the concepts. This study reviews in detail the first and second concepts whereas not the third because the first and second concepts often implicitly employed the third in explaining their concepts of sustainability.

\subsection{Sustainability as Continued Services and Benefit Flows}

Brinkerhoff (1994) summarized sustainability as 'sustainable flows of ongoing benefits from development investment after the initial investment period' (Brinkerhoff, 1994). It was partly derived from the concerns for deleterious impacts of short-term program implementation during the 1980s (Brinkerhoff, 1994). As a result, recipient countries and international donors started shifting their attention to sustainability defined as the long-term continuation of program 
benefits (Brinkerhoff, 1994). Esselman (1994) identified sustainability as 'ability of benefit flows to be maintained after project funding ceased' (Esselman, 1994). Yet, the author noted that sustained benefits do not imply the program continuation per se, but continued benefits through beneficiaries themselves, non-governmental organizations (NGOs), community groups or host government after the initial investment (Esselman, 1994). Additionally, the author mentioned donors may need to sustain benefits over a longer period of time particularly for disadvantaged or marginalized populations (Esselman, 1994). Levinger and McLeod (2002) argued 'provision of the service itself is no longer necessary because the benefits have been demonstrated and the recipients will demand, purchase or use the service' emphasizing an aspect of self-reliance. While conceptualizing sustainability as continued benefit flows, Brown (1998) specified the concept by including: continued local actions stimulated by the program, other services and initiatives resulted from program-built capacity, and program-initiated goods and services delivered and maintained five years after donor resources terminated (Brown, 1998). Although there is little consensus on sustainability timeframe as in one, three or five years, sustainability should be measured not only beyond the program completion but at multiple time points to capture variations over time due to its dynamic nature (Stirman et al., 2012).

International health programs tended to highlight the desired health outcomes of programs to address the initial problems whereas the concept of sustainability remains as the continued benefits. For instance, Pallas et al. (2013) defined sustainability as 'continued use of program components and activities for the continued achievement of desirable program and population outcomes' (Pallas et al., 2013). Rasschaert et al. (2014) stated sustainability is 'capacity to maintain program services at a level that will provide ongoing prevention and treatment for a health problem after termination of major financial, managerial and technological assistance from an external donor' (Rasschaert et al., 2014). Focusing on transition process of health programs, Bao et al. (2015) noted 'a well-managed transition process should enable the sustained delivery of program services hence, sustained health outcomes even if there may be changes in who delivers services or the mechanisms through which they are provided' (Bao, Rodriguez, Paina, Ozawa, \& Bennett, 2015).

The central concept of sustainability as continued benefit flows seems little changed with slight modifications over time. However, more complex and dynamic concepts have been emerged, reflecting evolution of aid programs and shifting attention towards interacting environment that influence programs.

\subsection{Sustainability as a Continuous Evolution of Interactive Factors and External Environments}

Evolution of aid programs prompted multidimensional concepts of sustainability (Gruen et al., 2008). In addition, diverse research traditions take different views on this complex concept (Gruen et al., 2008). Mog et al. (2004) stated sustainability is 'inherently difficult to specify because its specific meaning and practical applications are dynamic, indefinite and contested': dynamic due to shifting background, indefinite as a result of abstract, context-specific and long-term goals, and contested from many human values, perceptions and competing political interests (Mog, 2004). This intricate concept stresses dynamic natures of sustainability 'driven by interactions between stakeholders, institutions and beneficiaries, and ensured continuation through adaptations to broader environments, which consequently requires attention to broader organizational settings and system dynamics' (Chilundo, Cliff, Mariano, Rodríguez, \& George, 2015). With this emerging concept, sustainability in a child survival (Sarriot et al., 2004) and HIV program (Walsh, Mulambia, Brugha, \& Hanefeld, 2012) was identified 'contribution to the development of conditions enabling individuals, communities, and local organizations to express their potential, improve local functionality, develop mutual relationships of support and accountability, and decrease dependency on insecure resources' (Sarriot et al., 2004). The resources mentioned by Sarriot et al. (2004) include financial, human, technical, informational resources for local stakeholders to pursue health and development beyond project interventions (Sarriot et al., 2004).

Overall, the multidimensional concept of sustainability is difficult to capture in a simple manner. In a more comprehensive manner, Gruen et al. (2008) summarized sustainable programs as 'ones functioning as a complex system with the interacting key components such as initial problems, program elements and their drivers in important ways with one another, all of which are situated in a particular context with mostly defined resource availability' (Gruen et al., 2008). Stirman et al. (2012) similarly implied this complexity as 'elucidating the ways in which influences interact to enhance or challenge sustainability will ultimately facilitate an understanding from a complex-systems perspective and may also lead to the development of strategies to promote sustainability in contexts and circumstances in which certain factors are absent or less than optimal' (Stirman et al., 2012). In sum, conceptualizing sustainability may require exploring its interactive factors in a particular contextual setting in a specific manner. The following sections examine the 11 contributing factors identified for sustainability.

\section{Contributing Key Factors to Sustainability}

The key factors were identified with their perceived meanings as described in each paper. Table 3 summarizes the key 11 factors that constructed sustainability models and appeared at least three times across the papers. 
Table 3. Key factors that constructed sustainability models are summarized with number of papers in which the factors were identified

\begin{tabular}{|c|c|c|}
\hline Factors $*$ & No. of papers ${ }^{1}$ & Publication \\
\hline Capacity building ${ }^{2}$ & 16 & All 16 papers reviewed \\
\hline Political will, commitment & 10 & $\begin{array}{l}\text { (Bao et al., 2015), (Bossert, 1990), (Brinkerhoff, 1994), (Esselman, } \\
\text { 1994), (Chilundo et al., 2015), (Gruen et al., 2008), (Mog, 2004), (Pallas } \\
\text { et al., 2013), (Rasschaert et al., 2014), and (Sarriot et al., 2004) } \\
\text { (Bao et al. 2015), (Bossert 1990), (Brown. 1998) (Chilundo et al. }\end{array}$ \\
\hline New or continuous funding source & 8 & $\begin{array}{l}\text { 2015), (Esselman, 1994), (Levinger \& McLeod, 2002), (Sarriot et al., } \\
\text { 2004), and (Walsh et al., 2012) }\end{array}$ \\
\hline Community participation & 6 & $\begin{array}{l}\text { (Bossert, 1990), (Esselman, 1994), (Gruen et al., 2008), (Mog, 2004), } \\
\text { (Pallas et al., 2013), and (Rasschaert et al., 2014) }\end{array}$ \\
\hline Linkage, connectedness & 5 & $\begin{array}{l}\text { (Brinkerhoff, 1994), (Chilundo et al., 2015), (Levinger \& McLeod, } \\
\text { 2002), (Sarriot et al., 2004), and (Walsh et al., 2012), }\end{array}$ \\
\hline Socio-culture $^{3}$ & 5 & $\begin{array}{l}\text { (Bossert, 1990), (Brinkerhoff, 1994), (Mog, 2004), (Pallas et al., 2013), } \\
\text { and (Rasschaert et al., 2014) }\end{array}$ \\
\hline Program effectiveness ${ }^{4}$ & 5 & $\begin{array}{l}\text { (Bossert, 1990), (Brinkerhoff, 1994), (Chilundo et al., 2015), (Esselman, } \\
\text { 1994), and (Hanh et al., 2009) }\end{array}$ \\
\hline Institutionalization & 3 & (Bennett et al., 2011), (Esselman, 1994), and (Hanh et al., 2009) \\
\hline $\begin{array}{l}\text { Transition and taking-over } \\
\text { responsibility }\end{array}$ & 3 & $\begin{array}{l}\text { (Bao et al., 2015), (Levinger \& McLeod, 2002), and (Rasschaert et al., } \\
\text { 2014) }\end{array}$ \\
\hline Negotiation & 3 & (Bossert, 1990), (Esselman, 1994), and (Rasschaert et al., 2014) \\
\hline Communication & 3 & (Bao et al., 2015), (Chilundo et al., 2015), and (Rasschaert et al., 2014) \\
\hline
\end{tabular}

\footnotetext{
*: Identified and mentioned as factors, components, elements, determinants, criteria or domains

1: When frequency is the same, it is not in the order of importance, but for more logical transition to the next factor

${ }^{2}$ : Capacity building covers across multiple stakeholders, not limited to program beneficiaries

3 : Socially or culturally acceptable or complying with local values, not easily controllable

4: Effectiveness as either perceived or real
}

The most frequently appearing factor was capacity building, cited by all 16 papers as a crucial feature of sustainability. However, this comes partly from the fact that capacity building indicated multiple, if not all, stakeholders in a program. Other factors that repeatedly appeared were; political will or commitment (10), new or continuous funding resource (8), community participation (6), linkage or connectedness (5), acceptance in socio-culture (5), program effectiveness (5), institutionalization (3), transition of responsibility (3), negotiation (3) and communication (3).

\subsection{Capacity Building}

All papers identified capacity building as one of their essential factors to sustain benefits. In some cases, the term, capacity building, may indicate a specific stakeholder such as a government, local organization, NGO, community and individual beneficiary while, in other cases, it is used in an abstract manner such as capacity building in decision-making, policy formulation, and human or social capital accumulation. For clarity, this study examines capacity building at two levels, 1) an institutional and organizational level, mainly relevant to central government, implementing organization, and national decision-making processes, and 2) a local-communities and individual-beneficiaries level. The boundary between the two levels is often not entirely distinct because responsible ministries, local communities, beneficiaries and donors may influence each other's capacity building.

\subsubsection{Organizational and Institutional Capacity}

Rasschaert et al. (2014) referred to organizational capacity as 'the means and conditions that are required to function independently and maintain the core activities' (Rasschaert et al., 2014). Organizational capacity for health programs may include progressive involvement of line ministries, integration of program activities in existing health services, availability of required resources, and flexibility to adapt to changing healthcare needs over time (Rasschaert et al., 2014). Esselman (1994) mentioned institutional strength as 'organizational and technical capacity of the cooperating ministries within the host governments, and institutional capacity of a recipient country is critical to sustain desired activities and benefits' (Esselman, 1994). Brown (1998) further stated 'sustainability indeed is about institutional capacity to generate a minimum level and quality of valued outputs over the long term, continued effectiveness, maintenance of an acceptable level of institutional capacity, and most importantly, converting that capacity into actual performance' (Brown, 1998). Yet, donors often insist on measuring performance with verifiable output indicators which may bear little relationship to institutional capacity (Brown, 1998). Brinkerhoff (1994) viewed capacity and performance as a cycle; they are integral to one another for sustainability, and one stimulates the other. The best way to 
build capacity, the author argued, is through 'actual performance by institutional learning and adaptation to produce benefits, and the best way to achieve performance is by building capacity' (Brinkerhoff, 1994). Additionally, increasing organizational capacity was considered vital in dealing with innovation, uncertainty and hostility for continued program performance (Brinkerhoff, 1994). Brinkerhoff and Goldsmith (1992) supported this view with their finding, a positive association between the strengthened organizational capacity and continuation of benefits beyond the project period (Brinkerhoff \& Goldsmith, 1992). Interestingly, Bossert (1990) suggested that governmental capacity to collect revenue and distribute it to programs may be a minimal requirement with little institutional corruption for sustained benefits, which thus makes building institutional capacity a major policy objective (Bossert, 1990). However, Esselman (1994) debated that as a sustainability criterion, institutional capacity may be impractical due to lack of good measures of the capacity. Institutional capacity, therefore, unlikely can be compared across countries or assessed over time in a single country (Esselman, 1994). On the other hand, proponents of institutional capacity may argue improved economic and social indicators with declining aid dependency is a prima facie case of adequate institutional capability (Esselman, 1994).

Overall, institutional and organizational capacity is necessary to convert resources into continued performance. Bennett et al. (2011) identified some key activities to promote governmental capacity such as systematic improvement in technical and managerial skills in staffs, development of training structure and materials, and establishment of government norms and guidelines among others (Bennett, Singh, Ozawa, Tran, \& Kang, 2011). Those above studies mostly looked institutional and organizational capacity at a national government level. Yet, others more focused on locally based organizations and NGOs. For local entities, organizational capacity indicates the capacity that needs to exist in local organizations in order to maintain essential services and activities (Sarriot et al., 2004). Sarriot et al. (2004), however, distinguished between organizational capacity and viability; significant efforts can be invested in capacity improvement of local NGOs' performance while actually increasing its dependency on international funding sources, thus potentially putting the NGOs' viability in question (Sarriot et al., 2004). With organizational capacity of NGOs and their staff, Chilundo et al. (2015) identified factors that can facilitate their capacity: developing operational guidelines and tools, training in a standardized manner, and supportive supervision and logistics.

\subsubsection{Capacity Building in Local Communities and Individual Beneficiaries}

If promoted changes are to be sustained over time, local people and their institutions should be responsible for sustaining the changes (Mog, 2004). Thus, sustainable programs must invest substantial resources in developing the local-social capital to maintain performance over a long term (Mog, 2004). This requires building and strengthening capacities of local communities and individual actors through basic education and extension, technology-transfer, networking and partnership-building, specialized training, and placing people toward future learning, adaptation and innovation (Mog, 2004). Pallas et al. (2013) in health programs identified training, rather than capacity building per se, as one of the enabling factors for sustainability (Pallas et al., 2013). The authors pointed that intensive training should be part of program design and management since training is essential to motivate heath workers for sustaining program benefits (Pallas et al., 2013). Levinger and McLeod (2002) mentioned capacity building warrants that local actors have the skills and resources needed to deliver high-quality services until those services achieve sustainable benefit flows. This reflects empowerment of the local actors by allowing them to take control of the process affecting their lives as well as programs (Levinger \& McLeod, 2002). In theory, program sponsors should not exit until the benefits of the program can be sustained, but in reality, it is difficult to recognize a point where local actors acquired capacity and resources to sustain benefits (Levinger \& McLeod, 2002). It in turn implies that a level of capacity in local actors can be a good proxy to measure program sustainability. Moreover, Bao et al. (2015) argued capacity assessment should help identify areas in need of investment and signal the amount of capacity building and training required for sustained services (Bao et al., 2015). When capacity is insufficient, capacity-building activities should begin developing the necessary components for continued delivery of program activities.

Political climates strongly influence the ability of communities and individuals to express their capacity (Sarriot et al., 2004). More capable and organized communities and individuals will exercise greater influence on social and political progress (Sarriot et al., 2004). The importance of recognizing the external influence including politics was similarly stressed because individuals or communities are not isolated from the external influences operating at national, regional, or global scales (McConville \& Mihelcic, 2007). Thus, program staffs should be aware of political influences, investigate their dynamics, and design interventions accordingly, even if a program has little control over political influences (McConville \& Mihelcic, 2007).

\subsection{Political Will and Commitment}

Aid programs are a product of policy choices by various groups at international, regional, national, and local levels (Brinkerhoff, 1994). Outcomes of such political dynamics shape programs' missions, intervention areas, scopes, and 
budgets (Brinkerhoff, 1994). Therefore, it is critical to pay special attention to policies that influence program activities and stakeholders. Equally important is recognizing that policies are essential sources of cues and incentives for stakeholder behaviors over the life of a program (Brinkerhoff, 1994). Accordingly, political commitment is identified as one of the main external factors to sustain benefits across the papers. In addition, political commitment relates itself to or results from institutional or governmental capacity. It should be mentioned use of the term 'political commitment' was often interchangeable with political advocacy, national commitment, and political support, will or engagement.

Esselman (1994) defined national commitment as 'consensus among important decision makers and interest groups that the program goals and objectives are a national priority'. McConville and Mihelcic (2007) broadened the concept of political commitment with the term 'political cohesion' stressing coordinated development efforts by the international community and local politicians for program success (McConville \& Mihelcic, 2007). Political cohesion, thus improves alignment of development programs with host country priorities and coordination of aid efforts at local, national, and international levels to increase ownership and efficient delivery of services (McConville \& Mihelcic, 2007). In other words, both domestic and global political environments are key to gain strong national commitment and prioritize program goals (Chilundo et al., 2015). In a broader sense, initial political support and commitment to programs should be continued through a series of national consultations and policies to reach domestic objectives and international goals as well as sustain the program (Chilundo et al., 2015). Mog (2004) identified political support as process- and outcome-oriented criteria to evaluate program sustainability. As a process-oriented criterion, political support was used to recognize the influence of external conditions while, as an outcome-oriented, to evaluate policies which promoted program goals or tailored interventions within an existing policy structure in a favorable manner (Mog, 2004). Political infrastructure was strongly associated with institutional strength to support programs and commit to budgets (Bossert, 1990). Different levels of political infrastructure with administrative routines, budgetary resources, and skilled officials may correspond to programs' variation in sustainability (Esselman, 1994). Signs for supportive policy environment may include a program being embedded in a national policy or specific program goals being reflected in national or subnational plans and budgets (Bao et al., 2015). However, existing policies can undermine program sustainability when they hinder effective program processes, which in turn should be a target for a policy change (Bao et al., 2015). Likewise, governmental supports to sustain programs are reflected in secured advocacy from political leaders, funding allocation, or rewards for program staffs (Pallas et al., 2013). Yet, challenges arise to secure political support when other programs compete financially or politically among policy decision-makers. Additionally, political will should address broader contextual components such as poverty or illiteracy to sustain programs' long-term benefits (Rasschaert et al., 2014). Ironically, to maintain national commitment and increase the likelihood of program sustainability, effective results need to be demonstrated at a relatively early stage of a program although focus on short-term impacts can be detrimental to achieve sustainability (Esselman, 1994).

One of the critical signals of political commitment is a degree of financial commitment. As research on sustainability frequently focuses on financial aspects, it is often assumed that programs will be sustained with national funding commitment (Bossert, 1990).

\subsection{New or Continuous Funding Resources}

Financing is probably the most frequently studied, though not necessarily the most significant factor influencing sustainability (Esselman, 1994), since the existence of secured and diversified funding often is vital to program sustainability (Bao et al., 2015). However, securing funding is complicated in other issues such as economic conditions, political will, national priorities, and institutional and organizational capacity. Heterogeneous donor landscapes may influence funding in that presence of generous donors can discourage the responsible entities of the program to accept financial duties (Bao et al., 2015).

The exit of implementing organizations frequently means the end of funding, sometimes with a short notice (Levinger $\&$ McLeod, 2002). Ideally, implementing organizations should mobilize adequate resources to maintain a necessary scope and high-quality services for sustained benefit flows from the initial phase of a program (Levinger \& McLeod, 2002). After a program ends, financial resources may include funding from other external donors, community resources, public contribution from local or national government, private sector resources, and introduction of fees-for-services (Levinger \& McLeod, 2002). Box 1 summarizes potential funding resources in healthcare programs (Esselman, 1994). 
Box 1. Four funding resources after donor funding ends modified from Esselman (1994)

User fees: System charges beneficiaries of healthcare for a portion of cost. Community, individual care provider, government, or professional organization can set fees. Success of fee-for-service programs depends on beneficiaries' willingness and ability to pay, availability of alternative service delivery systems, and perceived quality and effectiveness of services.

Incorporating private sector: Private healthcare providers include midwives, traditional healers, private physicians and others who sustain themselves without government assistance. System may achieve full self-financing by solving issues of employee incentives, cost controls, and cross-subsidies.

Efficiency: Improved efficiency lowers cost, including efficient institutional arrangements to expand coverage through health volunteers or para-professional health workers etc.

Endowments: Endowment ensures a steady financial source to support long-term planning for health services.

One of the most prevalent financing mechanisms is national absorption of program costs; after a donor funding stops, national budgetary commitment is a cost recovery mechanism (Bossert, 1990). Thus, program sustainability depends on whether these funding resources were developed during the program life; when national budgets progressively absorbed recurrent program costs, the programs were more likely to be sustained than those that were externally funded at high levels throughout the program life (Bossert, 1990). Heavy reliance on external funding threatens sustainability when external funding declines because heavy reliance on it creates uncertainties, increases a risk of attrition, and prevents programs from scaling up and harmonizing with other programs (Chilundo et al., 2015). To encourage recipient governments to devote more resources for program funding stability, vertical partnerships and policy dialogues need to be established despite challenging factors such as national debts, weak economic conditions and other competing public sectors (Esselman, 1994).

Two papers paid particular attentions to beneficiaries' responsibilities to secure funding as a main financial resource (Bao et al., 2015; Levinger \& McLeod, 2002). When self-financing of the program is necessary, several prerequisites may arise: presence of transparent management of budgets and expenditures, sufficient levels of already-secured funding, and adequate transfer of financial responsibilities from the donor (Bao et al., 2015). One of the most promising means for self-financing is a user-fee system. To establish this system, generating demands for high-quality services are essential (Levinger \& McLeod, 2002). However, introducing user fees can create a form of price discrimination to exclude the intended beneficiaries, and threaten the program objective to reach the most needed (Brown, 1998). To solve these issues, setting a fee as low as a token, or a tiered fee structure based on income levels was suggested (Brown, 1998). During the funded period, service delivery is not vulnerable to deterioration (Walsh et al., 2012). After financial support ceased though, service levels in quality and quantity may drop resulting in decreased demand, consequently uncertainty in program funding. Although none of the reviewed papers specified characteristics of service quality, Olsen (1998) indicated in healthcare systems that integral characteristics of sufficient quality include appropriateness, efficiency, equity, effectiveness, acceptability, accessibility and affordability (Olsen, 1998). Funding uncertainties may deteriorate some of these quality characteristics. It could be unrealistic for donors to make certain activities self-financing in deprived beneficiaries or underfunded governments (Esselman, 1994). Sarriot et al. (2004) also cautioned impractical expectation that health services can fully self-finance in a country that depends on foreign aid at a national level (Sarriot et al., 2004).

In sum, secured and stable funding is one of the critical factors for sustainability. High dependence on external donors, insufficient national budget, inadequate service in quality and quantity, and resulting low demands are identified threats to continued benefit flows. Walsh et al. (2012) indicated a program that is most likely sustain itself financially is one that is emerged from a community, engaging itself with a supportive community structure, and working with existing local and national level systems (Walsh et al., 2012).

\subsection{Community Participation}

Broad-based community participation can be fundamental to most effective sustainable programs (Mog, 2004). Community participation is a process that fosters empowerment and ownership in community members through their direct participation in decision-making that affects the community (McConville \& Mihelcic, 2007). Rasschaert et al. (2014) identified four essential elements to reinforce community participation: common geographical areas, interests, feelings of ownership, and social relationships (Rasschaert et al., 2014). Also, there are false assumptions about community; communities are homogeneous, community leaders act in the best interest of their people, governmental and local workers share the same goal (Rifkin, 1986). Rifkin (1986) disputed these assumptions in that communities often are not homogenous because individual concerns can override the community goals, community leaders might use new opportunities to enrich themselves, and governmental staffs may mobilize the community resources for other national programs (Rifkin, 1986). 
One of the two approaches, a top-down or bottom-up approach, initiates community participation (Rifkin, 1986). The two approaches make different assumptions; the top-down intends to solve existing problems within a short time period while the bottom-up leans toward making changes in a longer term (Rifkin, 1986). Activities from the top-down involve a community as a passive means to reach program objectives (Rasschaert et al., 2014). Similarly, Levinger and McLeod (2002) argued, within the top-down framework, community participation is viewed as a means to an end (Levinger \& McLeod, 2002). On the other hand, the bottom-up focuses more on empowerment of the community, providing it with active voices in decision-making, program implementation, and planning activities (Rasschaert et al., 2014). The top-down was not successful in the past while the bottom-up can be more effective and acceptable as it is often based on mutual trust and respect (Rasschaert et al., 2014). When constructing community participation from the bottom-up, key consideration should be beneficiaries' own motivation and need, thus an essential first step is creating a platform where communities can voice their needs (Rasschaert et al., 2014). This platform can later serve as an information feedback loop to adapt program activities responding to changing needs. Two studies related community participation to funding resources for continued program activities because community participation can be crucial part of a cost-recovery mechanism (Bossert, 1990; Esselman, 1994). Effective community participation enhances sustainability by forming constituency that demands continued program activities (Bossert, 1990). Yet, the beneficiaries' demand was less critical in countries where the national budget provided funding to sustain program activities, than in countries where a community-based cost-recovery mechanism was a major form of funding. In the latter countries, community participation was linked to the beneficiaries' willingness to pay for services (Bossert, 1990).

Overall, to create sustained benefit flows, local communities need to be viewed as collaborators and lead actors in program decision-making processes (Mog, 2004) while unsustainable programs diminish community trust and support (Gruen et al., 2008). However, Rifkin (1986) argued the inputs and outputs of community participation are hard to measure under heavy cultural, historical, political, and economic influences. Moreover, community participation is a dynamic process with a constant state of changes, rather than static; people in the same program over time with more experiences may not hold the same definition of community participation now as they did then (Rifkin, 1986).

\subsection{Linkage and Connectedness}

Part of a strategic planning process of a program should devote to building supportive networks and alliances among stakeholders (Brinkerhoff \& Goldsmith, 1992). Two studies stated 'strategic relationships or connectedness are critical for organizational viability to achieve organizational missions due to their interdependence in a given institutional environment' (Sarriot et al., 2004; Walsh et al., 2012). A program's strategic partnership among line ministries helps establish key relationships to define program goals, directions and strategies while partnerships between line ministries, NGOs and communities help mobilize and motivate beneficiaries in planning process (Chilundo et al., 2015). Partnerships, thus, should be established among vertical and horizontal organizations, and among local actors themselves (Levinger \& McLeod, 2002). Local networks can provide resilience, information and structured support, and mitigate risks as international donors phase out (Levinger \& McLeod, 2002). At an individual level, identifying and connecting actual and potential leaders in local organizations lay the ground for a successful program exit as this process ensures these actors understand the program goals, and are more likely committed to their roles (Levinger \& McLeod, 2002). Box 2 describes five practices of how stakeholders engage themselves for sustainable partnerships.

Box 2. Practices for sustainable partnerships, modified from Levinger \& McLeod (2002)

Commit to clear goals that have been established with inputs from all partners

Consider diverse types of organizations, including those from the nongovernment, corporate, and public sectors, as potential partners

Allow and encourage partners to specialize for benefitting comparative advantage of each actor

Use diversity within partnership to respond to changing trends in and emerging threats from external environment

Use partnership for complex programs, rather than simple projects, so that benefits of complementary skills and resources outweigh costs of coordinating partnership

\subsection{Socio-culture}

Socially and culturally acceptable programs are based on local traditions or core values, which are uncontrollable and embedded in a broader context (McConville \& Mihelcic, 2007). Also, sociocultural consideration of a program integrates the program into an existing fabric of society and local community (McConville \& Mihelcic, 2007). The sociocultural factor should weigh more when programs are implemented within a variety of cultural settings and beliefs (McConville \& Mihelcic, 2007). A program model built on cultural values and social habits creates strong social links and networks between members to obtain long-term results (Rasschaert et al., 2014). Rifkin (1986) pointed out culture 
and social structures can heavily influence community participation. Thus, assessment of how a program plays sociocultural roles within the community is important because it may determine community's willingness to maintain the services (McConville \& Mihelcic, 2007). Cultural acceptance of program goals such as promoted behavior changes, and of methods such as technologies and policies applied can play an important role to sustain a program and its benefits (Mog, 2004).

\subsection{Program Effectiveness}

Effective programs are those that have achieved expected goals and objectives with a relatively efficient use of resources (Bossert, 1990). When viewed effective, programs gain broader support, hence more likely to secure funding than ones considered otherwise (Bossert, 1990). Often, reputation for effectiveness, rather than empirical evidence, seems significant for program sustainability (Bossert, 1990). One reason is reputation generates favorable decisions by officials, service providers and beneficiaries regardless of whether a scientific evaluation backs the reputation (Bossert, 1990). Esselman (1994) likewise identified program effectiveness, perceived or reputed so, as one of the key factors to influence program sustainability by influencing decisions from all stakeholders in program activities even if concrete evidence on effectiveness lacked (Esselman, 1994). Program effectiveness for sustainability needs to be carefully understood, in that while showing program effectiveness at program's earlier stages can maintain national commitment to increase the likelihood of program's survival, focus on short-term impacts is detrimental to longer-term sustainability (Esselman, 1994).

\subsection{Institutionalization}

Program institutionalization was mainly connected to political and organizational decision-making, and linkage. Bennett et al. (2011) identified institutionalization as 'integration of key elements of the program into organizational procedures, and behaviors of government agencies and other main implementing partners' (Bennett et al., 2011). The authors also categorized institutionalization into three levels. First, at the most basic level, routinization needs to occur; key practices of a program should be adapted to the government system, and implemented on a routine basis. As the next higher level, program practices should be reflected in government norms, standard operating procedures, guidelines, and policies. The highest level of program institutionalization provokes dynamic changes within a broader government system and stimulates interactions, which is important for system-wide changes over time (Bennett et al., 2011).

Success in sustaining benefits requires alignment of technical and managerial aspects of a program with the government norms and standards (Bennett et al., 2011). Or, programs can be integrated into existing hierarchical structures of host country's line ministries and local agencies, which necessitates building wide support from administrators interested in continuing the program (Esselman, 1994). Integrated programs draw several different players into the system, and potentially spread the positive notion of continuing program activities (Esselman, 1994). Although this strategy probably complicates decision making, prospects for program sustainability can be higher (Esselman, 1994). However, embedment of a program in a line ministry may increase program's vulnerability due to system's own constraints such as weak supervision or poor logistics among others (Chilundo et al., 2015).

\subsection{Transition of and Taking over Responsibility}

During a program transition, responsibilities for program and staff management, financing, monitoring and evaluation, and other administrative tasks must be efficiently transitioned (Bao et al., 2015). The most effective exit strategy arranges key program elements for a timely planned transition of new roles and responsibilities to local actors (Levinger $\&$ McLeod, 2002). And, it is critical to ensure individuals internalize their responsibilities in the program and logistics with new attitudes and behaviors (Levinger \& McLeod, 2002). Taking over responsibilities is closely related to capacity of individuals, and a degree of community participation; community participation unites capable people with common needs to take more responsibilities (Rasschaert et al., 2014). When individual beneficiaries progressively take more responsibilities in service delivery, they are perceived as a valuable aid for a program rather than mere beneficiaries (Rasschaert et al., 2014). However, who carry program responsibilities often remains unclear because those carrying responsibilities need incentives such as official recognition, remuneration and accountability, which is related to motivation (Rogers \& Coates, 2015). Therefore, monitoring motivation is necessary as the communities and individuals can suffer 'participation fatigue' to maintain their motivation for carrying new responsibilities (Rasschaert et al., 2014). Assessment of capacity may be required to gauge a level of training needed for successful transition of responsibility (Bao et al., 2015). However, as a transition process involves inevitable shifts in power and authority, it can face stakeholder resistance (Bao et al., 2015); including new people in decision-making involves a power shift disrupting existing power monopoly, and likely results in a range of conflicts (Rifkin, 1986). 


\subsection{Communication}

Communication helps align expectations, seek common goals, and build positive relationships among key stakeholders (Bao et al., 2015). Good communication is fundamental to strategic planning, and vital to ensuring sustainability (Bao et al., 2015). During the program implementation phase, communication among stakeholders can facilitate dissemination of key information, and coordinate program activities (Chilundo et al., 2015). National-level communication is particularly important because ineffective communication may cause government's failure to provide required resources for the program (Chilundo et al., 2015). Also important is efficient communication in order to gain high visibility of program activities and outcomes (Rasschaert et al., 2014). Fluent information flow from effective communication can increase perception and acceptance of the program, and promote needed changes to sustain benefits (Rasschaert et al., 2014). Reversely, when beneficiaries are little aware of the program, or doubt service quality caused by limited information flow, they are less likely to join or support the program (Rasschaert et al., 2014). Poorly coordinated communication for shifted priorities or changed values creates resistance to continued service (Bao et al., 2015). Thus, strengthening communication channels and their coordination promotes program sustainability, and generates synergies for sustained benefits. Effective communication channels can further ensure political support that helps stabilize program funding. Finally, synchronized communication channels smooth negotiation processes, which can foster stakeholders' motivation, resource mobilization and favorable policies for sustainability.

\subsection{Negotiation Process}

A mutually respectful negotiation involves give-and-take between stakeholders. This process can influence sustainability through how collaboration and compromise occur to support a program (Bossert, 1990). Esselman (1994) stated that when responsible officials of a recipient country viewed a program as being imposed by a donor agency, the program was less likely to be sustained than programs designed and approved by a mutually respectful negotiation between the two entities. With imbalanced negotiations, recipient government officials who felt excluded from program development may become opponents to the program (Bossert, 1990). Their opposition creates unnecessary resistance or lack of interest in supporting the program. On the other hand, programs that were designed with national participation in negotiation to fit into recipient countries' priorities were more likely to be sustained (Bossert, 1990). Therefore, aid policy should reinforce a mutually-benefitting negotiation process (Bossert, 1990). Additionally, at an individual level, negotiation and political skills in community workers can foster sustainable programs because their negotiation skills help motivate themselves for a positive career path, supportive supervision, stable supply of resources, and improved training programs (Chilundo et al., 2015).

\section{Concluding Remarks}

This study reviewed the select papers in the specified fields of international aid programs, and identified the frequently adopted concepts of sustainability and contributing factors. Sustainability is about continued effectiveness over time which requires the continual conversion of capacity into performance and constant adjustment to changes in both internal and external environments (Brown, 1998). Overall, the sustainability factors identified interconnect and precondition one another for synergistic effects. For instance, capacity building was the most cited factor to sustain benefits and services. However, all other factors, though with different degrees, strongly influence a level of capacity built or needed; in other words, successful capacity building relies on and requires other factors. At the same time, factor relations and interactions could be less clear due in part to nuances involved in a program's goal and nature (Chilundo et al., 2015). Moreover, external or contextual settings such as political climate, social structure, economic condition, donor dynamic and culture appeared significant as most of the reviewed papers mentioned them even though they did not separately identify them as sustainability factors. These broader contextual conditions may enable or disable other frequently appearing factors contributing to sustainability as no single program can control these external conditions.

More research is necessary to build empirical evidence on how and what factors work more closely to one another, and affect program activities, outcomes, impacts and finally sustainability in a certain setting. As mentioned previously, sustainability is inherently difficult to simplify due to its specific meanings and practical applications (Mog, 2004). Even so, careful analysis of factor relations may improve policy decision-making for more efficient international aid programs for long-term benefits. The current study found that not a sufficient number of studies has been conducted on sustainability models and factors in international aid programs of health, food or rural development. This may have resulted from the expected complications empirically to study sustainability with diverse program goals within heterogeneous contextual settings. Another difficulty was capturing nuanced meanings and wording across the select papers that had started with different research objectives and methods. However, these complications should not prevent the findings from being valid for or applicable to further studies to improve program sustainability. 


\section{Acknowledgements}

The author thanks Dr. Jennifer Coates and Dr. Beatrice Lorge Rogers at the Gerald J. and Dorothy R. Friedman School of Nutrition Science and Policy, Tufts University, Boston, U.S.A for generous advice to develop initial research directions and methods during the directed study and kind comments.

\section{Conflicts of Interest}

The author declares no conflicts of interest.

\section{References}

Bao, J., Rodriguez, D. C., Paina, L., Ozawa, S., \& Bennett, S. (2015). Monitoring and Evaluating the Transition of Large-Scale Programs in Global Health. Global Health: Science and Practice, 3(4), 591-605. https://doi.org/10.9745/ghsp-d-15-00221

Bennett, S., Singh, S., Ozawa, S., Tran, N., \& Kang, J. (2011). Sustainability of donor programs: evaluating and informing the transition of a large HIV prevention program in India to local ownership. Global health action, 4. https://doi.org/10.3402/gha.v4i0.7360

Bossert, T. J. (1990). Can they get along without us? Sustainability of donor-supported health projects in Central America and Africa. Social science \& medicine, 30(9), 1015-1023. https://doi.org/10.1016/0277-9536(90)90148-L

Brinkerhoff, D. W. (1994). Looking Out, Looking In, Looking Ahead. PA Times, 17(12), 11.

Brinkerhoff, D. W., \& Goldsmith, A. A. (1992). Promoting the sustainability of development institutions: A framework for strategy. World Development, 20(3), 369-383. https://doi.org/10.1016/0305-750X(92)90030-Y

Brown, D. R. (1998). Evaluating institutional sustainability in development programmes: Beyond dollars and cents. Journal of International Development, 10(1), 55-69. https://doi.org/10.1002/(SICI)1099-1328(199801)10:1<55::AID-JID452>3.0.CO;2-W

Chilundo, B. G., Cliff, J. L., Mariano, A. R., Rodríguez, D. C., \& George, A. (2015). Relaunch of the official community health worker programme in Mozambique: is there a sustainable basis for iCCM policy? Health Policy and Planning, 30(suppl 2), ii54-ii64. https://doi.org/10.1093/heapol/czv014

Esselman, J. (1994). Sustainability, Sustainable Development, and the Health Sector. Issues Paper(1).

Gruen, R. L., Elliott, J. H., Nolan, M. L., Lawton, P. D., Parkhill, A., McLaren, C. J., \& Lavis, J. N. (2008). Sustainability science: an integrated approach for health-programme planning. The Lancet, 372(9649), 1579-1589. https://doi.org/10.1016/S0140-6736(08)61659-1

Hagen-Zanker, J., \& Mallet, R. (2013). How to do a rigorous, evidence-focused literature review in international development, A Guidance Note. London: Overseas Development Institute.

Honadle, G., \& VanSant, J. (1985). Implementation for sustainability: Lessons from integrated rural development: Kumarian Pr.

Levinger, B., \& McLeod, J. (2002). Hello I must be going: ensuring quality services and sustainable benefits through well-designed exit strategies. Newton, MA: Education Development Center Inc.

McConville, J. R., \& Mihelcic, J. R. (2007). Adapting life-cycle thinking tools to evaluate project sustainability in international water and sanitation development work. Environmental Engineering Science, 24(7), 937-948. https://doi.org/10.1089/ees.2006.0225

Mog, J. M. (2004). Struggling with sustainability - a comparative framework for evaluating sustainable development programs. World Development, 32(12), 2139-2160. https://doi.org/10.1016/j.worlddev.2004.07.002

OECD. (1989). Sustainability in Development Programmes: A Compendium of Evaluation Experience: Organisation for Economic Co-operation and Development.

Olsen, I. T. (1998). Sustainability of health care: a framework for analysis. Health Policy and Planning, 13(3), $287-295$. https://doi.org/10.1093/heapol/13.3.287

Pallas, S. W., Minhas, D., Pérez-Escamilla, R., Taylor, L., Curry, L., \& Bradley, E. H. (2013). Community health workers in low-and middle-income countries: what do we know about scaling up and sustainability? American journal of public health, 103(7), e74-e82. https://doi.org/10.2105/AJPH.2012.301102

Rasschaert, F., Decroo, T., Remartinez, D., Telfer, B., Lessitala, F., Biot, M., . . Van Damme, W. (2014). Sustainability of a community-based anti-retroviral care delivery model - a qualitative research study in Tete, Mozambique (Vol. 17, pp. 18910): International AIDS Society. 
Rifkin, S. B. (1986). Lessons from community participation in health programmes. Health Policy and Planning, 1(3), 240-249. https://doi.org/10.1093/heapol/1.3.240

Rogers, B., \& Coates, J. (2015). Sustaining Development: A Synthesis of Results from a Four-Country Study of Sustainability and Exit Strategies among Development Food Assistance Projects. USAID.

Sarriot, E. G., Winch, P. J., Ryan, L. J., Bowie, J., Kouletio, M., Swedberg, E., . . . Pacqué, M. C. (2004). A methodological approach and framework for sustainability assessment in NGO - implemented primary health care programs. The International journal of health planning and management, 19(1), 23-41. https://doi.org/10.1002/hpm.744

Stirman, S. W., Kimberly, J., Cook, N., Calloway, A., Castro, F., \& Charns, M. (2012). The sustainability of new programs and innovations: a review of the empirical literature and recommendations for future research. Implementation Science, 7(1), 1.

Walsh, A., Mulambia, C., Brugha, R., \& Hanefeld, J. (2012). The problem is ours, it is not CRAIDS. Evaluating sustainability of Community Based Organisations for HIV/AIDS in a rural district in Zambia. Globalization and health, 8(1), 1. https://doi.org/10.1186/1744-8603-8-40

\section{Copyrights}

Copyright for this article is retained by the author(s), with first publication rights granted to the journal.

This is an open-access article distributed under the terms and conditions of the Creative Commons Attribution license which permits unrestricted use, distribution, and reproduction in any medium, provided the original work is properly cited. 